\title{
Exact Solution of Displacement of Tapered Interference Fit
}

\author{
Peng SHANG \\ School of Equipment Engineering, Engineering University of CAPF, China 710086 \\ sipone@163.com
}

Keywords: exact solution, displacement, taper, interference fit

\begin{abstract}
Tapered interference fits can avoid the influence of keyways on the parts strength and transfer large torques. In this paper, a model was developed to study the influence of the taper on the interference fit between a hub and a shaft. Using the classic elastic plane stress theory, the exact solutions of the displacements of the hub and shaft are derived. Then the calculation method of the magnitude of the tapered interference fit was presented. Finally taking a screw propeller system as an example, the above solutions were calculated by using the numerical method. The results show that the taper plays a key role in the interference fit. The present analytical solutions are expected to be useful in the structure design of tapered interference fits for hubs and shafts.
\end{abstract}

\begin{tabular}{|llll|}
\hline Nomenclatures & & \\
$E$ & elastic moduli [Gpa] & $r$ & radius [m] \\
$K$ & reciprocal of taper & $u$ & radial displacement [m] \\
$M$ & torque [N/m] & $\delta$ & magnitude of tapered interference fit [m] \\
$N$ & rotational speed [rpm] & $\varepsilon$ & strain \\
$R$ & outer radius of propeller hub [m] & $\mu$ & Poisson's ratio \\
$f$ & static friction coefficient & $\sigma$ & stress [Pa] \\
subscripts & & \\
$H \quad$ hub & $r$ & radial direction \\
$S \quad$ shaft & $S$ & small end \\
$b$ & big end & $\theta$ & tangential direction \\
\hline
\end{tabular}

\section{Introduction}

Interference fits are widely used in gear design for fixation of pinions, couplings, and the like on shafts. They can transfer large torques, are easy to produce and offer significant cost advantages [1]. Commonly a cylindrical interference fit is applied. Such a clearance between hub and shaft is chosen, that the stresses of all regions of the fit can be calculated by Lame's solution. The design and stress analysis of cylindrical interference fits are studied broadly and delicately, most of which are concerned with a laminated composite tube which is interference fitted onto other structure like a bearing bush or a hub. The model is developed as a hollow cylinder is subjected to internal or external pressure, depending on the relative location in the joint assembly and the elastic solution can be found for such a case in the literature [2-9]. But the solution of cylindrical interference fits is not very suitable for screw propeller systems because the propeller hub and shaft are connected together by tapered interference fit.

Accordingly, the aim of this paper is to develop a better calculation method used in the design of hubs and shafts for tapered interference fits which are affected by tapers. Using the classic elastic plane stress theory, the equations of the tapered interference fits are derived. Then the exact solutions of the displacements of the tapered interference fits are obtained. Finally taking the tapered interference fits of a screw propeller system as an example the above solutions are calculated and plotted. 


\section{Analytical model}

In this research, the structure analyzed here is only the part of the tapered interference fit of a screw propeller system. The tapered interference fit between the hub and shaft is shown in Fig. 1. The radiuses of the small end and big end of the shaft are indicated as $r_{s}$ and $r_{b}$ respectively. $R$ is the outer radius of the hub and $L$ is the length of the tapered interference fit. Further we stipulate that the elastic moduli $E$ and Poisson's ratio $\mu$ as well as the density $\rho$ of the hub and shaft are equal.
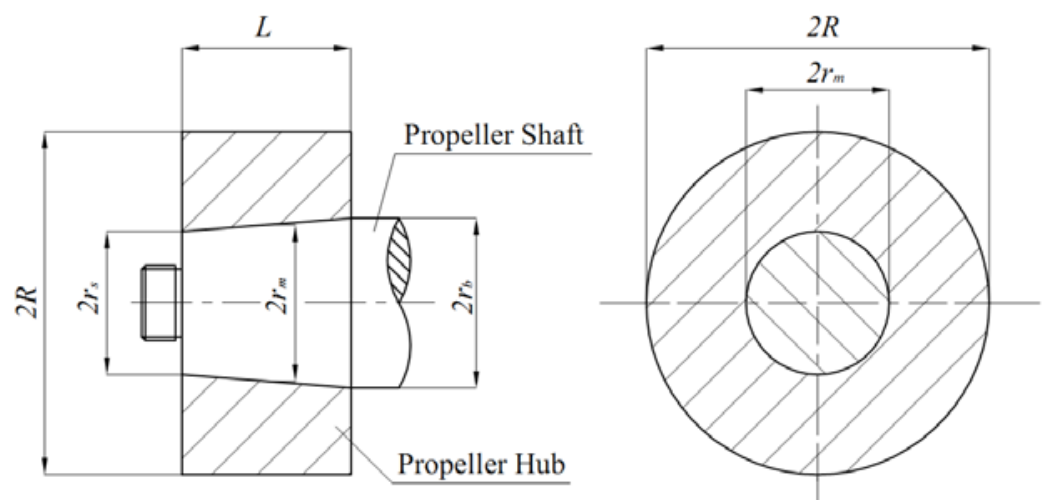

Fig. 1 The tapered interference fit between the propeller hub and shaft.

To study the connection characteristics for the tapered interference fit of the propeller hub and shaft, the influence of the taper $K$ on the displacements of the propeller hub and shaft are investigated as follow:

The radial stresses and tangential stresses of the propeller hub and shaft were given in the literature [10].

$$
\begin{gathered}
\sigma_{r, H}=-\frac{2 M}{\pi f\left(r_{b}-r_{s}\right)\left[4 R^{2}-\left(r_{s}+r_{b}\right)^{2}\right]} \cdot \frac{R^{2}-r^{2}}{K r^{2}}, \\
\sigma_{\theta, H}=\frac{2 M}{\pi f\left(r_{b}-r_{s}\right)\left[4 R^{2}-\left(r_{s}+r_{b}\right)^{2}\right]} \cdot \frac{R^{2}+r^{2}}{K r^{2}}, \\
\sigma_{r, S}=-\frac{2 M}{\pi f\left(r_{b}-r_{s}\right)\left(r_{s}+r_{b}\right)^{4}} \cdot \frac{\left(r_{s}+r_{b}\right)^{2}-4 r^{2}}{K}, \\
\sigma_{\theta, S}=-\frac{2 M}{\pi f\left(r_{b}-r_{s}\right)\left(r_{s}+r_{b}\right)^{4}} \cdot \frac{\left(r_{s}+r_{b}\right)+4 r^{2}}{K} .
\end{gathered}
$$

The strain-displacement relation and constitutive equation can be given as

$$
\begin{aligned}
& \varepsilon_{r}=\frac{d u}{d r}, \quad \varepsilon_{\theta}=\frac{u}{r}, \\
& \sigma_{r}=E \varepsilon_{r}+\mu \sigma_{\theta}, \sigma_{\theta}=E \varepsilon_{\theta}+\mu \sigma_{r} .
\end{aligned}
$$

Combine Eqs. 1-6, the radial displacements of the propeller hub and shaft deduce to Eqs. 7-8 respectively,

$$
\begin{aligned}
& u_{H}=\frac{2 M r}{\pi f K E\left(r_{b}-r_{s}\right)} \cdot \frac{(1+\mu) R^{2}+(1-\mu) r^{2}}{\left[4 R^{2}-\left(r_{s}+r_{b}\right)^{2}\right] r^{2}}, \\
& u_{S}=-\frac{2 M r}{\pi f K E\left(r_{b}-r_{s}\right)} \cdot \frac{1-\mu}{\left(r_{s}+r_{b}\right)^{2}} .
\end{aligned}
$$

The magnitude of the tapered interference fit of the propeller hub and shaft can be given in Eq. 9 . $\delta=2\left|u_{H}-u_{S}\right|_{r=\left(r_{s}+r_{b}\right) / 2}$. 


\section{Numerical analysis}

This section studies the influence of the taper $K$ on the displacements of the propeller hub and shaft. The invariable parameters used in the interference fit are given in Table. 1.

Table 1. Invariable parameters of the interference fit

\begin{tabular}{cccc}
\hline parameters & values & parameters & values \\
\hline$E$ & $2.1 \times 10^{11}[\mathrm{pa}]$ & $f$ & 0.12 \\
$M$ & $85 \times 10^{3}\left[\mathrm{~N} \cdot \mathrm{m}^{3}\right]$ & $r_{b}$ & $251.5[\mathrm{~mm}]$ \\
$\mu$ & 0.3 & $r_{s}$ & $226[\mathrm{~mm}]$ \\
$R$ & $507.5[\mathrm{~mm}]$ & &
\end{tabular}

The influence of the taper $K$ on the displacement of the hub and shaft are shown in Fig. 2 and Fig. 3. The displacements of the hub and shaft both decrease with the growing of the taper $K$. The displacement of the shaft $u_{S}$ increases as the radius $r$ increases. However, the displacement of the hub $u_{H}$ is negatively correlated with the radius $r$, and it is always greater than zero. The maximum of the displacement of the hub and shaft both occur on the contact surface of the interference fit.

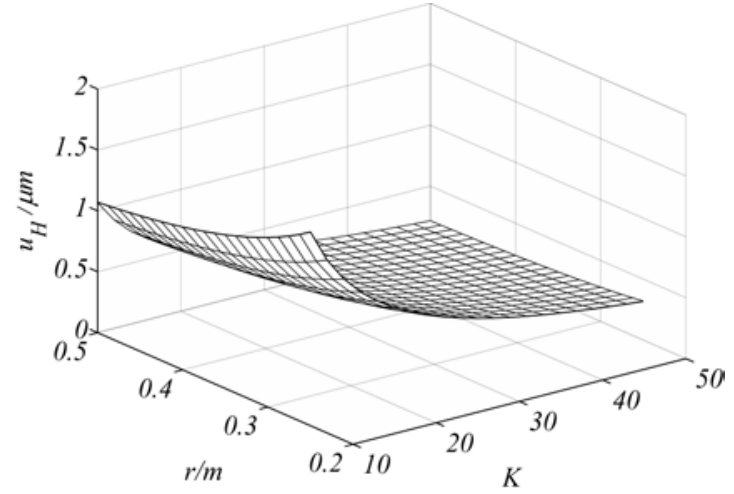

(a) The displacement of the hub.

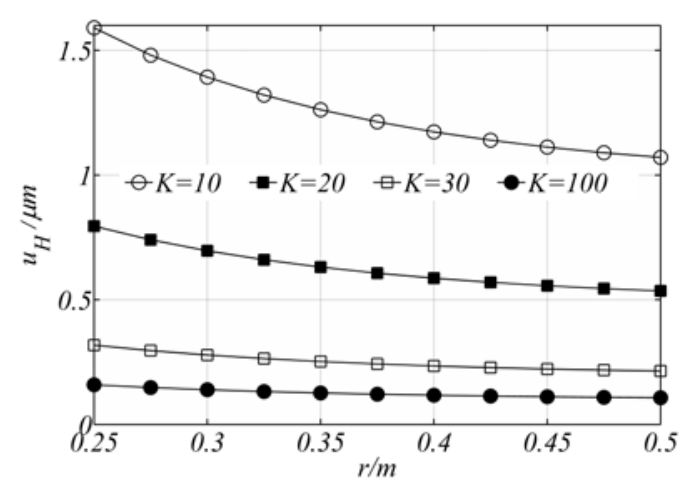

(b) Dependence of $u_{H}$ upon $K$.

Fig. 2 Influence of the taper on the displacement of the propeller hub.

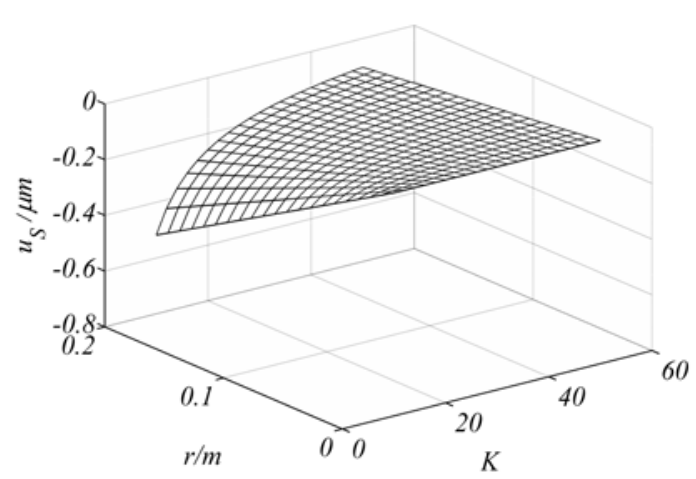

(a) The displacement of the shaft.

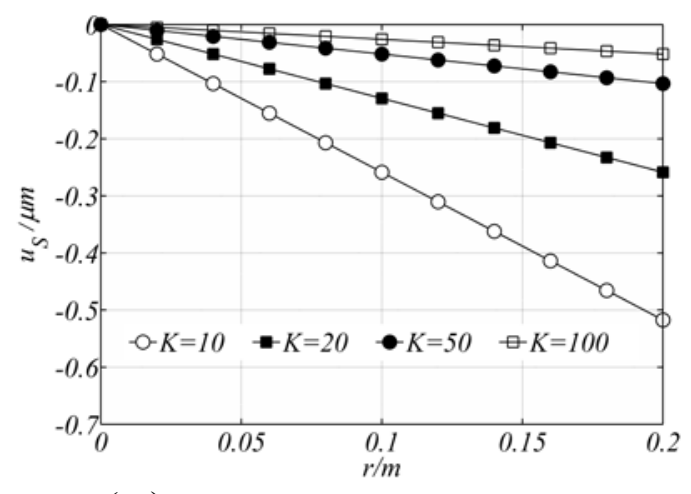

(b) Dependence of $u_{s}$ upon $K$.

Fig. 3 Influence of the taper on the displacement of the propeller shaft.

Fig. 4 shows the influence of the taper $K$ on the magnitude of the interference fit. The magnitude of the interference fit $\delta$ is positively correlated with the taper $K$. The influence of the taper $K$ on the magnitude of the interference fit $\delta$ is significantly for $K<10$. 


\section{Conclusions}

As a determine parameter, the taper have to be considered for the design of the tapered interference fit between the hub and shaft. The magnitudes of the radial stresses and tangential stresses of the propeller hub and the shaft are negatively correlated with the taper. The maximum magnitude of the displacement of the hub or shaft are related to the structure of the interference fit. Improving the strength of the contact surface of the hub and shaft is an effective approach to increase the torque transmission capacity.

Considering of the yield stress of the material, the selection of the taper of the interference fits has to be optimized. For this purpose further studies have to be performed.

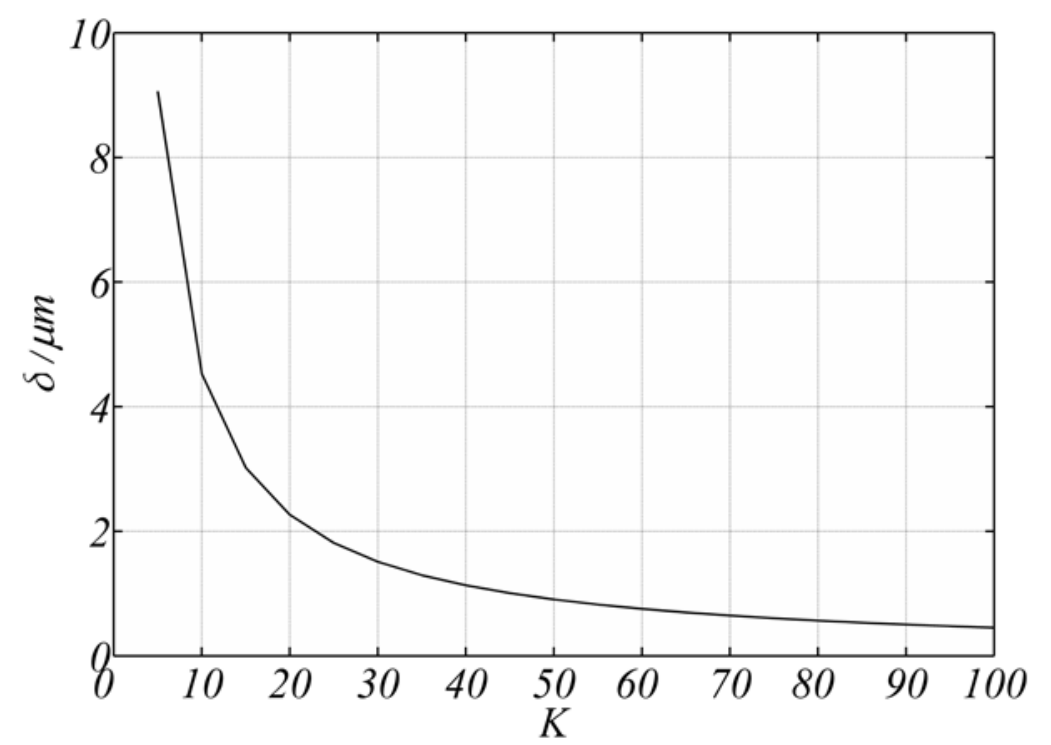

Fig. 4 Influence of the taper on the magnitude of the interference fit.

\section{References}

[1] F. G. Kollman: Journal of Mechanical Design Vol. 103 (1981), p. 61

[2] S.Sen and B.Aksakal: Materials and Design Vol. 25 (2004), p. 407-417

[3] D. Castagnetti and E. Dragoni: IMechE, J. Strain Analysis Vol. 40 (2005), p. 177-184

[4] Peng Shang, Ying Zhan, Sheng Feng et al, Advanced Materials Research Vol. 744 (2013), p. 180-184

[5] M. M. Calistrat and G. G. Leaseburge, in: presented at the Amer. Soc. Mech. Eng. Conf. (1974).

[6] Seung Woo Lee and Dai Gil Lee: Composite Structures Vol. 78 (2007), p. 584-595

[7] Pierre-Daniel Pfister and Yves Perriard, in: Proc. 8th ICEMS, 3054-3059, Wuhan, China (2008)

[8] Guoping Wang, Hualing Chen, Shemiao Qi et al, in: 3rd International Conference on Machinery, Materials Science and Engineering Applications, Wuhan, China (2013)

[9] Ling Xiong, Peng Shang, Youlin Xu, Applied Mechanics and Materials Vol. 423 (2013), p. 1438-1443

[10] Dincer Bozkaya, Sinan Müftü. Journal of Biomechanical Engineering Vol. 126 (2004), p. 393-401

[11] Xiaoqing Zhao, Peng Shang, Applied Mechanics and Materials Vol. 556-562 (2014), p. 4284-4287

[12] Robert W. Bosley, U.S. Patent 5,697,848. (1997)

[13] Guoping Wang, Hualing Chen, Shemiao Qi et al, in: 3rd International Conference on Machinery, Materials Science and Engineering Applications, Wuhan, China (2013)

[14] LI Weijian, Pan Cunyuan. Journal of Mechanical Strength Vol. 33 (2011), p. 86-92

[15] Dincer Bozkaya, Sinan Müftü. Journal of Biomechanics Vol. 36 (2003), p. 1649-1658

[16] YUAN Rongjuan. Journal of Machine Design Vol. 20, 2 (2003), p. 42-43 